\title{
La turistificación en el Centro Histórico de Oaxaca
}

\section{Mabel Yescas Sánchez}

Resumen. En este trabajo se analiza la evolución de la turistificación en el Centro Histórico de Oaxaca. Tres aspectos son abordados: la identificación de las relaciones existentes entre actores sociales y gubernamentales: municipal, estatal y federal; el comportamiento de la dinámica turística en la ciudad; y la exposición de los efectos que su desarrollo genera, en el nivel físico y socioespacial. Se plantea una modificación del discurso que sustenta la actividad turística, inicialmente enmarcada por la política federal de volverla motor de desarrollo de la ciudad, por una concepción de rescate económico y afluencia turística derivada de los hechos sociales de 2006 con el desalojo de maestros del centro. El empleo de estrategias metodológicas permitió la definición de etapas en el análisis, un antes y un después del conflicto magisterial, a fin de corroborar lo señalado en discursos y contrastar eventuales resultados después de una década de los problemas sociales.

Palabras clave: turistificación, Centro Histórico, patrimonialización, Oaxaca, transformación socioespacial.

•Profesora en la Universidad Autónoma Benito Juárez de Oaxaca. Correo-e: mabel.yescas@ gmail.com 


\section{The «touristification» of the Historic Center in Oaxaca}

Abstract. This work examines the evolution of the «touristification» in the Historic Center in Oaxaca. Three elements are involved in the identification of the existing relations between social actors and those in government: municipal, state and federal; the patterns evident in the tourist dynamic in the city; and the apparent effects that arise from its development, at the fiscal and socio-spatial levels. It calls for a change in the discourse that drives tourism activity, initially supported by federal policy intending to use it as an engine of development for the city, to an initiative of economic recovery via tourist flows following the 2006 demonstrations that ended in the forced displacement of teachers from the Center. The use of methodological strategies allowed for the definition of analytical stages, a «before» and an «after» of the teacher's conflict, that which was called for in public speeches and contrasted with the situation following a decade of social struggle.

Keywords: touristification, Historic Center, heritage development, Oaxaca, socio-spatial transformation. 


\section{Introducción}

Para la literatura, la actividad turística se entiende como un proceso social de alcance internacional, con efectos locales y viceversa, asociado a fenómenos económicos globales. Rodolfo Bertoncello (2006) identifica dos momentos en su existencia, uno vinculado a un sistema industrial fordista, el turismo de masas, diversificado por áreas y gustos, en el que impera un Estado altamente intervencionista y un mercado interno desarrollado; otro, el actual, relacionado con un modelo neoliberal, caracterizado por una sociedad de consumo, un Estado debilitado y un mercado abierto. En esta etapa la actividad deja de ser geográficamente polarizada para convertirse en algo más difuso. Los tiempos y espacios del turismo cambiaron (Córdoba, 2009) y se privilegió un turismo de estancias cortas, cada vez más orientado hacia áreas urbanas (Hiernaux, 2014).

En el sistema neoliberal el turismo cultural cobra importancia. La nostalgia es la motivación, mientras que los bienes históricos son la atracción. El patrimonio es el elemento que sintetiza una identidad y una cultura, y los turistas acaban siendo espectadores interesados en conocer algo auténtico con qué conectarse con el pasado, susceptible de ser valorado, disfrutado a través de su consumo. Por medio de un proceso de turistificación, los centros históricos latinoamericanos parecen o creen poder encontrar una opción a la crisis económica que atraviesan, al atraer el turismo nacional y aprovechar los cambios observados a escala mundial en términos de preferencias turísticas, que miran a la cultura como motor de desarro1lo (De Sicilia, 2012). En este ensayo se entiende como turistificación a un proceso de desarrollo turístico organizado y voluntarista de un espacio y el resultado del mismo. 
La inserción de los centros históricos mexicanos a una lógica turística patrimonialista ha implicado que los gobiernos locales se enfoquen en la ejecución de intervenciones físicas y simbólicas para el mejoramiento de imagen de sus centros; en ocasiones las instancias públicas son acompañadas por el sector privado para el desarrollo de proyectos específicos. Unas intervenciones consisten en renovaciones y rehabilitaciones de estructuras físicas o del espacio público, el mantenimiento de infraestructura y del equipamiento urbano; otras, en medidas de control y vigilancia de la sociedad local y de los visitantes a partir de diversas acciones represivas de los sectores sociales más desfavorecidos, mediante lo que se ha bautizado como «higienización», como lo señalan Hiernaux y González (2015). Ambos tipos de intervenciones han sido cuestionados en la literatura por los efectos que conllevan, tanto transformaciones espaciales como impactos sociales y segregación urbana, mayor expulsión de la población de bajos ingresos de los centros (la llamada «gentrificación»), entre otros. Hasta la fecha, los estudios enfocados al desarrollo de la actividad turística en tales espacios evidencian también una baja revitalización económica, no compensada con la cantidad de recursos económicos que los gobiernos locales destinaron por décadas (González, 2014).

El objetivo del presente trabajo es entonces indagar cómo ha evolucionado la turistificación del Centro Histórico de Oaxaca hasta el año 2015. Primero se identifican las relaciones existentes entre actores, asociaciones y organizaciones civiles, y gobierno de los tres ámbitos — municipal, estatal y federal - en este proceso; luego, se analiza la dinámica turística en la ciudad; por último, se exponen los efectos que su desarrollo genera en el nivel físico y socioespacial.

Se emplearon metodologías cualitativas y cuantitativas. Respecto al componente cualitativo, se efectuó una revisión bibliográfica detallada, se 


\section{La tURistificación en el Centro Histórico de OAXACA}

utilizaron fuentes hemerográficas, y se elaboraron y aplicaron entrevistas semiestructuradas. Desde lo cuantitativo, se realizó la búsqueda, la depuración y el procesamiento de datos estadísticos vinculados al tema turístico; cuando fue necesario se llevó a cabo un análisis cartográfico. Lo que a continuación se expondrá es el resultado del examen de la información recabada desde 2015, que continúa construyéndose para desarrollar la tesis de doctorado. La aplicación de las diversas técnicas mencionadas permitió definir dos etapas claras del actuar público y privado en el proceso turistificador: un antes y un después de la profunda crisis social que se suscitó en la capital oaxaqueña en 2006.

El concepto de turistificación guiará el desarrollo de la investigación. Se entiende por aquel la apropiación de la ciudad a través del tiempo por el turismo; o como lo definen Ana Lía del Valle Guerrero y Silvana Soledad Gallucci (2015), un proceso mediante el cual se construye un territorio turístico en vista de su valoración económica, expresada en una ocupación creciente de la infraestructura hotelera y de alojamiento en general, así como un uso más intenso de los equipamientos para visitantes; también implica una polarización creciente de flujos turísticos y efectos diversos en términos socioeconómicos para la ciudad visitada (Navarrete, 2017).

La literatura advierte que dicho fenómeno se sostiene en dos componentes: el primero es la atractividad, que consiste en la existencia de lugares, rasgos, atributos susceptibles de ser consumidos por el turismo; el segundo es la autenticidad de objetos, símbolos, algunos de ellos fabricados específicamente para el turismo. Ambos determinan el patrimonio que sustenta un espacio turístico. A la fase de selección y activación de elementos histórico-culturales donde intervienen distintos actores asociados a la práctica turística se le conoce como patrimonialización (Bertoncello, 2006); ésta suele 
ser consecuencia de miradas externas, en específico de la Organización de las Naciones Unidas para la Educación, la Ciencia y la Cultura (UNESCO) y el Consejo Internacional de Monumentos y Sitios (Icomos), además de valoraciones locales de los gobiernos municipal, estatal y federal, con el respaldo de ciertos sectores de la sociedad civil (Pérez, 2013). La patrimonialización y la turistificación son ampliamente estudiadas desde múltiples disciplinas: sociología, antropología, economía, geografía humana y arquitectura, entre otras (Navarrete, 2017; Hiernaux y González, 2015; Martínez, 2015; De Sicilia, 2012; Córdoba, 2009; Troncoso y Almirón, 2005).

\section{Hacia la consolidación de la actividad turística del Centro Histórico de Oaxaca}

La actividad turística de la ciudad inicia en la era porfiriana, cuando se registraron los primeros trabajos para su florecimiento. Dos obras fueron las principales causantes del despegue turístico: la apertura ferroviaria ${ }^{1} \mathrm{y}$ la restauración del templo de Santo Domingo (Lira, 2014). ${ }^{2}$ Posterior a la Revolución, la situación financiera de Oaxaca se mostraba en crisis, ello repercutió en el estado de los edificios históricos, pues no se invirtió en su conservación; en esa época el gobierno municipal sólo se enfocaba en el mantenimiento de plazas y jardines. Fue entre 1928 y 1932, debido a

${ }^{1}$ Su objetivo era facilitar el flujo comercial. Para ese periodo Oaxaca era ya un nodo regional de la economía del sureste mexicano en el tema mercantil; después el ferrocarril propició la llegada de personas por intereses turísticos.

${ }^{2}$ La finalidad era atraer viajeros que desearan conocer las bellezas arquitectónicas de Oaxaca. Más tarde la ciudad empezó a registrar flujos crecientes de visitantes, lo que incentivó la construcción de mesones y hoteles. 


\section{LA tURistificación en el Centro Histórico de OAXaca}

los sismos que azotaron a Oaxaca, cuando las administraciones gubernamentales se volcaron en su recuperación, mientras descuidaban otras tareas encaminadas al desarrollo urbano, actitud que perduró hasta entrada la década de 1960. Resultaba menos costoso, en efecto, recuperar que construir nuevos edificios, lo que ocasionó una presión hacia el reciclaje de edificios coloniales y decimonónicos.

A partir de la dinámica turística estadounidense, que tuvo lugar después de la Segunda Guerra Mundial con el deseo de explorar nuevos territorios, entre ellos el latinoamericano, el gobierno federal promovió programas cuyo objetivo era el fomento turístico en el nivel estatal. Producto de la adopción de esa política en Oaxaca, el gobierno estatal emprendió también diversas acciones, en algunos casos en compañía de agentes empresariales. El papel del sector privado en esta etapa estuvo enfocado a su consolidación mediante asociaciones civiles, fungió como promotor turístico a escala local, participó en la profesionalización del personal turístico y asesoró a comerciantes con perfil turístico. Por ejemplo, en 1930, el gobierno local y representantes del sector privado instauraron un Comité Local Pro Turismo. ${ }^{3}$ Una de las primeras tareas que afrontó el Comité fue la difusión de la cultura estatal por distintos medios y formas. ${ }^{4}$ Para 1942, en la ciudad de Oaxaca, se celebró la Primera Gran Feria Indígena Estatal; ${ }^{5}$ su éxito incitó

${ }^{3}$ Integrado por representantes de varios sectores: económico, comercio, industria, agricultura, banca, medios de transporte y comunicaciones, propietarios de hoteles, entre otros (Lira, 2014). El propósito era legitimar las decisiones sobre promoción turística, pero añadiéndole el tema de identidad.

${ }^{4}$ Se apoyó en informes generados durante la administración previa, del exgobernador Genaro V. Vásquez, que manifestó el interés de exponer la cultura oaxaqueña. Asimismo, en 1941, con el aumento a la difusión del potencial turístico de la ciudad mediante folletos y tarjetas, se llegó a trascender internacionalmente.

${ }^{5}$ Se expuso la diversidad étnica existente en la entidad, observada por turistas nacionales y extranjeros, quienes quedaron seducidos por la presencia indigena. Lira (2014) indica que tal 
al gobierno estatal a activar otros temas culturales, además de la ya conocida Guelaguetza. ${ }^{6}$ En la década de 1960 el gobierno reforzó el impulso turístico desde vertientes disímiles: constitución de asociaciones civiles, elaboración de planes normativos, compendios de información, oferta de acceso a fondos, etcétera (Lira, 2014) (cuadro 1). Esa actitud perduró hasta los 1990 e incluso distintos medios de comunicación hicieron hincapié en la creciente polarización social que padecía la entidad. ${ }^{7}$

\section{Cuadro 1}

Acciones emprendidas para fomentar el turismo en el estado (continúa)

\begin{tabular}{c|lc}
\multicolumn{1}{c}{ Década } & \multicolumn{1}{c}{ Acción } & \multicolumn{1}{c}{ Actores } \\
\hline 1960 & $\begin{array}{l}\text { Patronato estatal de turismo } \\
\text { Semana del oaxaqueño ausente }\end{array}$ & Gobierno estatal \\
& $\begin{array}{l}\text { Leyendas que acompañan } \\
\text { las festividades de la Guelaguetza }\end{array}$ & Gobierno estatal
\end{tabular}

momento fue crucial en la definición de la imagen de la ciudad. Contemplar en un espacio histórico, tipo colonial, una cultura indígena, expresada de múltiples formas (folclor, gastronomía, vestuario, dialectos, por referir algunos), festejada cada año con una Guelaguetza, posibilitó creer en la resistencia de los indígenas sobre la imposición de valores occidentales en tiempos de la Conquista. Imaginario cuestionable, puesto que ese indigenismo que aún persiste en un mundo moderno sugiere más un acto de conveniencia gubernamental por la atracción turística generada que el resguardo de una cultura e identidad.

${ }^{6}$ Se realizó por vez primera el 25 de abril de 1932 para conmemorar los 400 años de la fundación de la ciudad. Es un espectáculo dancístico regional, actualmente de fama internacional, promocionado como fiesta de origen prehispánico y escenificado por representantes de comunidades indígenas.

${ }^{7}$ No se inscriben cambios en los niveles socioeconómicos de la mayor parte de la población después de décadas de inversión a la actividad turística.

${ }^{8}$ Sólo funcionó en la administración que la implementó. Se desarrollaba en la última semana del mes de mayo como fiesta en honor de los oaxaqueños radicados fuera de la ciudad. La intención era que los oaxaqueños alejados de su población de origen pudieran encontrar al volver un territorio pintoresco, pero insertado en la lógica moderna. 


\section{La tURistificación en el Centro Histórico de OAXaca}

\section{Cuadro 1}

Acciones emprendidas para fomentar el turismo en el estado (conclusión)

\begin{tabular}{|c|c|c|}
\hline Década & Acción & Actores \\
\hline \multirow{3}{*}{1970} & $\begin{array}{l}\text { Manual de información } \\
\text { turística del estado }\end{array}$ & $\begin{array}{l}\text { Gobiernos estatal } \\
\text { y federal }\end{array}$ \\
\hline & $\begin{array}{l}\text { Consejo de Turismo } \\
\text { del Estado de Oaxaca9 }\end{array}$ & $\begin{array}{l}\text { Representantes } \\
\text { de sectores } \\
\text { económicos } \\
\text { ligados al turismo }\end{array}$ \\
\hline & Consejo de Fomento Turístico del Estado & Gobierno estatal \\
\hline \multirow{3}{*}{1980} & $\begin{array}{l}\text { Patronato para la preservación } \\
\text { del patrimonio histórico } \\
\text { del estado de Oaxaca }\end{array}$ & $\begin{array}{l}\text { Gobiernos estatal } \\
\text { y municipal }\end{array}$ \\
\hline & $\begin{array}{l}\text { Especialidad en turismo } \\
\text { niveles bachillerato y licenciatura }\end{array}$ & Gobierno estatal \\
\hline & $\begin{array}{l}\text { Celebración del rango } \\
\text { de ciudad por } 450 \text { años }\end{array}$ & Gobierno estatal \\
\hline \multirow{6}{*}{1990} & $\begin{array}{l}\text { Fondo mixto de promoción } \\
\text { turística de ciudades coloniales }\end{array}$ & $\begin{array}{l}\text { Gobiernos estatal } \\
\text { y federal }\end{array}$ \\
\hline & $\begin{array}{l}\text { Plan de acción para la zona } \\
\text { de desarrollo turístico prioritario }\end{array}$ & Gobierno estatal \\
\hline & $\begin{array}{l}\text { Consejo consultivo turístico } \\
\text { del estado de Oaxaca }\end{array}$ & Gobierno estatal \\
\hline & Instituto Oaxaqueño de las Culturas & Gobierno estatal \\
\hline & $\begin{array}{l}\text { Red de Unidades Ecoturísticas } \\
\text { en Valles Centrales }\end{array}$ & Gobierno estatal \\
\hline & $\begin{array}{l}\text { Organización Estatal de Productores } \\
\text { de Artesanos del estado de Oaxaca }\end{array}$ & Gobierno estatal \\
\hline
\end{tabular}

Fuente: elaboración propia apoyada con informes de gobierno y Lira (2014).

${ }^{9}$ Su meta era elevar, vigorizar, dignificar y aprovechar al máximo la industria turística de la entidad. 
El gobierno estatal, en esa fase, fue el actor que más acciones desarrolló; el sector privado, mediante la constitución de asociaciones, en ocasiones lo acompañó y en otros momentos lo presionó para la ejecución de tareas concretas: profesionalización del turismo, inversión en la promoción turística, flexibilidad normativa en los usos de suelo, entre otras labores.

\section{Cuadro 2}

Obras emblemáticas emprendidas por el gobierno para fomentar el turismo en la ciudad

\begin{tabular}{c|ll}
\multicolumn{1}{c}{ Año } & \multicolumn{1}{c}{ Obra } & \multicolumn{1}{c}{ Función } \\
\hline \multirow{1}{*}{1962} & $\begin{array}{l}\text { Fuente de las Siete Regiones }{ }^{10} \\
\text { Modernización } \\
\text { de un edificio porfiriano }\end{array}$ & $\begin{array}{l}\text { Promover las distintas } \\
\text { regiones del estado }\end{array}$ \\
\hline $1964 \begin{array}{l}\text { Compra y remodelación } \\
\text { del Convento de los Siete Príncipes } \\
\text { de Correos }\end{array}$ & $\begin{array}{l}\text { En sus inicios fines artesanales, } \\
\text { actualmente casa de cultura }\end{array}$ \\
\hline 1986 & $\begin{array}{l}\text { Compra y readaptación } \\
\text { de la Casa de Cortés }\end{array}$ & $\begin{array}{l}\text { Museo de Arte } \\
\text { Contemporáneo }\end{array}$ \\
\hline \multirow{2}{*}{1980} & $\begin{array}{l}\text { Restauración del exconvento } \\
\text { del Carmen Alto }\end{array}$ & $\begin{array}{l}\text { Iglesia y Registro Civil } \\
\text { oaxaqueño }\end{array}$ \\
\hline $\begin{array}{l}\text { Peatonalización de calles } \\
\text { aledañas al zócalo, Macedonio } \\
\text { Alcalá y primera calle de 5 de mayo }\end{array}$ & Movilidad turística o recreativa \\
\hline
\end{tabular}

Fuente: elaboración propia apoyada con informes de gobierno y Lira (2014).

${ }^{10}$ Reubicada en 1964, hoy llamada de las Ocho Regiones, por el reconocimiento a la sierra Sur. 


\section{LA tURistificación en el Centro Histórico de OAXaca}

Varias obras acompañaron el despliegue de las actividades culturales, destacaron aquellas que registraron altos costos y fueron cuestionadas por la ciudadanía (cuadro 2). En el lapso de ocho décadas, 1920-2000, los gobiernos estatal y municipal implementaron más de cuatrocientos proyectos; de éstos, 199 fueron obras de restauración, arreglo y mantenimiento; el resto, 213, fueron obras nuevas (Lira, 2014).

\section{Giro al discurso turístico: rescate de la actividad}

Hasta entrada la primera década del tercer milenio, la política turística estatal mantuvo su trayectoria, planteaba aumentar la afluencia hacia la zona con el fin de posicionar a la ciudad entre los destinos turísticos más visitados del país; sin embargo, los hechos sociales registrados en 2006 transtornaron la tendencia positiva previa de manera muy intensa y modificaron la misión de las autoridades. ${ }^{11}$ El argumento común entre representantes de distintas empresas y organizaciones fue que el flujo de turistas decreció de la noche a la mañana. Tal situación afectó el alojamiento y toda la actividad económica local por el cierre de negocios, lo que generó pérdidas económicas severas;

${ }^{11}$ El 14 de junio se intentó desalojar al magisterio que tenía tomado el zócalo de la ciudad. Los disturbios se prolongaron hasta finales de ese año. Si bien el motivo nunca fue explícito, las actividades asociadas a la Guelaguetza estaban próximas, por lo cual debía presentarse la imagen de una ciudad bella, segura y tranquila, principalmente del centro, el espacio más visitado por nacionales y foráneos. Ante tal agresión la respuesta fue la constitución de la Asamblea Popular de los Pueblos de Oaxaca (APPO) cuyas demandas eran: reformas político administrativas en la asignación de recursos, mejor manejo de la justicia y de los procesos electorales y, principalmente, destitución del gobernador Ulises Ruiz (Sorroza, 2008). Se exhibió un descontento generalizado en la población hacia el mandatario por múltiples razones, entre ellas: su llegada con poca credibilidad a la gubernatura, además de la forma arbitraria en la que implementaba obras, caracterizada por un autoritarismo subnacional (Martínez, 2008). 
se planteó entonces la necesidad de un rescate urgente de la actividad turística. Los gobiernos estatal y municipal en este panorama se apropiaron del discurso empresarial y redefinieron estrategias para recuperar la afluencia turística; actuaron en diferentes ámbitos (económico, físico, social, cultural y político), se apoyaron mutuamente en varios momentos y favorecieron a la iniciativa privada y a determinados actores sociales. En este periodo resaltan tres segmentos que no habían sido utilizados como atractivos turísticos: crecimiento de la celebración de congresos y convenciones; aprovechamiento del turismo gastronómico; celebración de bodas y diversos eventos sociales, ahora emblemáticos en la promoción de la ciudad.

Por el lado político-económico, a raíz de los problemas sociales ocurridos en el centro de la ciudad, entes públicos y sociales intervinieron para recobrar la estabilidad social y económica: particularmente los tres niveles de gobierno (municipal, estatal y federal), así como Alfredo Harp Helú, personaje que desde la década de 1990 mostraba interés por Oaxaca, actitud catalogada por algunos como altruista y por otros como cacicazgo. ${ }^{12}$ La desemejanza entre el antes y el ahora en su actuar parece radicar en el grado de injerencia en la toma de decisiones estatales, las cuales no sólo se ubican en los ámbitos cultural y deportivo sino también en aspectos políticos y sociales, haciéndolo de manera abierta (cuadro 3) (Emeequis, 2016).

${ }^{12}$ Exbanquero que después de un secuestro se retiró en buena medida de la vida económica-financiera mostrando una actitud diferente, sobre todo benefactora con su estado de origen (véase Mateo, 2017). 


\section{La tURistificación en el Centro Histórico de OAXaca}

\section{Cuadro 3}

Acciones político-económicas emprendidas por diversos actores para recuperar el turismo en la ciudad

\begin{tabular}{|c|c|c|}
\hline$A \tilde{n} o$ & Acción & Actores \\
\hline 2006 & Obtención de recursos federales ${ }^{13}$ & Conaculta \\
\hline 2007 & Gestión de recursos ante federación ${ }^{14}$ & Fundación Alfredo Harp Helú \\
\hline 2008 & $\begin{array}{l}\text { El municipio es apoyado con } \\
2 \text { por ciento de sus gastos }\end{array}$ & Fundación Alfredo Harp Helú \\
\hline 2014 & $\begin{array}{l}\text { Se implementan descuentos } \\
\text { a cámaras empresariales } \\
\text { y se fomenta la regulación comercial }\end{array}$ & Gobierno municipal \\
\hline
\end{tabular}

Fuente: elaboración propia con datos de informes de H. Ayuntamiento de Oaxaca de Juárez (1976-2015).

De manera general, las acciones estuvieron encaminadas a incrementar la promoción del turismo religioso y cultural; ${ }^{15}$ asimismo, se incursionó en otros segmentos de mercado, como el de congresos y convenciones. También se intensificó y diversificó la promoción de la ciudad en medios publicitarios. Se apoyó la asistencia de artesanos a ferias de corte nacional e internacional. Coordinado por las cámaras empresariales, ${ }^{16}$ inició un

${ }^{13}$ Oaxaca se quedó con la mayor proporción de recursos destinados a las ciudades patrimonio: 83 millones de pesos.

${ }^{14}$ Alfredo Harp Helú se propuso como facilitador del Estado ante altos funcionarios, acción que le valió un reconocimiento municipal en 2008.

${ }^{15}$ Como se refleja en la procesión del silencio, el aniversario de la ciudad, la leyenda de la princesa Donají en la Guelaguetza, la fiesta de muertos y la Noche de Rábanos.

${ }^{16}$ Básicamente dos asociaciones manejan el tema: la Cámara Nacional de la Industria de Restaurantes y Alimentos Condimentados (Canirac) y la Asociación Mexicana de Hoteles y Moteles de Oaxaca AC. 
programa de capacitación de calidad a servidores turísticos que a la fecha continúa. Además, se ampliaron los hermanamientos entre ciudades mexicanas y extranjeras, y se llevaron a cabo programas vinculados al valor patrimonial (cuadro 4). En esta fase destaca nuevamente la participación activa de la Fundación Alfredo Harp Helú Oaxaca (FAHHO), con la implementación de programas relacionados con atractivos turísticos.

\section{Cuadro 4}

Acciones turísticas emprendidas por varios actores para recuperar la actividad en la ciudad (continúa)

\begin{tabular}{|c|c|c|}
\hline$A \tilde{n} o$ & Acción & Actores \\
\hline 2007 & Ampliación de las conexiones aéreas ${ }^{17}$ & $\begin{array}{l}\text { Gobierno estatal } \\
\text { e iniciativa privada }\end{array}$ \\
\hline \multirow[t]{2}{*}{2009} & $\begin{array}{l}\text { Elaboración de guía gastronómica } \\
\text { en internet }\end{array}$ & $\begin{array}{l}\text { Universidad Anáhuac } \\
\text { y gobierno municipal }\end{array}$ \\
\hline & $\begin{array}{l}\text { Implementación del programa } \\
« \text { Patrimonitos } »^{18}\end{array}$ & $\begin{array}{l}\text { FAHHO, } \\
\text { SEP-Oaxaca }\end{array}$ \\
\hline \multirow[t]{2}{*}{2010} & Implementación del Plan Reto ${ }^{19}$ & $\begin{array}{l}\text { FAHHO } \\
\text { y gobierno municipal }\end{array}$ \\
\hline & $\begin{array}{l}\text { Comienzan a financiarse congresos } \\
\text { y convenciones, también se impulsa } \\
\text { la gastronomía con el patrocinio de ferias }\end{array}$ & $\begin{array}{l}\text { Gobiernos municipal } \\
\text { y estatal }\end{array}$ \\
\hline
\end{tabular}

${ }^{17}$ Sumándose a las ya trazadas, Oaxaca-Guadalajara-Tuxtla Gutiérrez y Mérida.

${ }^{18}$ Tenía la finalidad de fomentar entre los niños el valor patrimonial cultural tangible e intangible, se mantuvo por varios años.

${ }^{19} \mathrm{Su}$ objetivo era consolidar el turismo cultural en la ciudad. 


\section{La turistificación en el Centro Histórico de OAXaca}

\section{Cuadro 4}

Acciones turísticas emprendidas por varios actores para recuperar la actividad en la ciudad (conclusión)

\begin{tabular}{|c|c|c|}
\hline $2012^{20}$ & $\begin{array}{l}\text { Se apoya la instalación } \\
\text { de exposiciones plásticas }\end{array}$ & $\begin{array}{l}\text { Instituto de Investigacio- } \\
\text { nes Estéticas-UNAM, Mu- } \\
\text { seo de los Pintores Oaxa- } \\
\text { queños, Casa de la Cultura } \\
\text { Oaxaqueña y gobierno } \\
\text { municipal }\end{array}$ \\
\hline \multirow{2}{*}{2014} & Se impulsa el turismo de bodas & Gobierno municipal21 \\
\hline & Se impulsa el turismo LGBT & Gobierno municipal22 \\
\hline
\end{tabular}

Fuente: elaboración propia con datos de informes de H. Ayuntamiento de Oaxaca de Juárez (1976-2015).

En cuanto al mejoramiento de imagen y conservación patrimonial, fueron varias obras y acciones desarrolladas por los gobiernos estatal y municipal entre 2006 y 2015. Las dos administraciones estatales que trataron el tema de la recuperación, las de Ulises Ruiz (2004-2010) y Gabino Cué (2010-2016), impulsaron de manera general trabajos de conservación y rehabilitación a iglesias; intervención a plazuelas, plazas, jardines; y embellecimiento de barrios como Jalatlaco, Xochimilco, ex Marquesado y Trinidad de las Huertas. Además, las administraciones citadas se hicieron cargo del mantenimiento constante de los espacios públicos: suministro y colocación de luminarias, mejoramiento de alumbrado público,

\footnotetext{
${ }^{20}$ En este mismo año el municipio de Oaxaca ganó el premio Tótem, como la mejor publicidad exterior en la categoría de turismo.

${ }^{21}$ Se volvió facilitador de permisos necesarios para la tradicional calenda-verbena.

${ }^{22}$ En 2015 el municipio recibió el premio al mejor diseño de producto turístico para dicho segmento.
} 
rehabilitación de banquetas y guarniciones, entre otras obras. En distintos momentos algunos empresarios patrocinaron acciones de rehabilitación, como es el caso de las fuentes históricas. Por ejemplo, en 2012 el Ayuntamiento firmó un convenio con la Universidad Regional del Sureste AC, los dos colegios de arquitectos del estado de Oaxaca y el Instituto Nacional de Antropología e Historia (INAH), para rescatar y preservar 43 inmuebles catalogados que se encontraban en condiciones de alto riesgo.

Convergieron dos lógicas en la administración de Ulises Ruiz. Una fue la descentralización de actividades gubernamentales formulada desde su llegada a la gubernatura, lo cual dio pauta a la conversión de usos de edificios históricos a fines turísticos y recreativos, como ocurrió con la Cámara de Diputados, la Casa Oficial y el Palacio de Gobierno. Otra línea de intervención fueron las obras encaminadas al mejoramiento de imagen de la ciudad. En su mayoría las tareas fueron cuestionadas por la población, debido a la falta de justificación para su desarrollo y los montos sobre los que se trabajaron, pero principalmente por la forma en que se ejecutaron; no existió consulta ciudadana en su diseño. Respecto a la administración de Gabino Cué, los proyectos apuntaron sólo al mejoramiento de imagen. No existió tanta controversia como con su antecesor, pero sí reticencia en los montos aplicados (cuadro 5). 


\title{
La tURistificación en el Centro Histórico de OAXaca
}

\author{
Cuadro 5 \\ Obras emblemáticas emprendidas \\ por el gobierno para recuperar el turismo (continúa)
}

\begin{tabular}{|c|c|c|}
\hline$A \tilde{n} o$ & Obra & Función \\
\hline \multirow{3}{*}{2005} & $\begin{array}{l}\text { Adecuación de la Cámara } \\
\text { de Diputados }\end{array}$ & Ahora Teatro Juárez \\
\hline & Rehabilitación de la Casa Oficial & $\begin{array}{l}\text { Hasta } 2010 \text { fue centro } \\
\text { de formación turística, } \\
\text { pero en } 2011 \text { recuperó su uso }{ }^{23}\end{array}$ \\
\hline & $\begin{array}{l}\text { Remodelación del zócalo } \\
\text { y paseo Juárez «El Llano» }\end{array}$ & Espacios públicos \\
\hline \multirow{2}{*}{2006} & $\begin{array}{l}\text { Rehabilitación del Palacio } \\
\text { de Gobierno }\end{array}$ & $\begin{array}{l}\text { Hasta } 2016 \text { fue museo, }{ }^{24} \\
\text { recientemente recuperó su uso }\end{array}$ \\
\hline & $\begin{array}{l}\text { Instalación de parquímetros } \\
\text { en el centro }\end{array}$ & $\begin{array}{l}\text { Para regular el uso } \\
\text { del espacio público } 25\end{array}$ \\
\hline \multirow[t]{2}{*}{2007} & $\begin{array}{l}\text { Ampliación a cuatro carriles } \\
\text { de la carretera que cruza el cerro } \\
\text { del Fortín }\end{array}$ & $\begin{array}{l}\text { Facilitar la accesibilidad } \\
\text { a la ciudad }\end{array}$ \\
\hline & $\begin{array}{l}\text { Mantenimiento al Auditorio } \\
\text { Guelaguetza }\end{array}$ & Centro de espectáculos \\
\hline 2008 & $\begin{array}{l}\text { Remodelación de la Fuente } \\
\text { de las Ocho Regiones }\end{array}$ & $\begin{array}{l}\text { Promover las distintas } \\
\text { regiones del estado }\end{array}$ \\
\hline 2010 & $\begin{array}{l}\text { Mejoramiento de imagen } \\
\text { del Mercado IV Centenario }\end{array}$ & $\begin{array}{l}\text { Centro de abastecimiento } \\
\text { barrial }\end{array}$ \\
\hline
\end{tabular}

${ }^{23}$ Gobierno del estado de Oaxaca (2011).

${ }^{24} \mathrm{~A}$ la fecha no existe alguna propuesta para reubicar las exposiciones (García, 2017).

${ }^{25} \mathrm{El}$ objetivo era darle preferencia a los visitantes en la ocupación del espacio; sin embargo, en el marco de las protestas magisteriales los parquímetros fueron destruidos. 


\section{Cuadro 5}

Obras emblemáticas emprendidas

por el gobierno para recuperar el turismo (conclusión)

\begin{tabular}{|c|c|c|}
\hline Año & Obra & Función \\
\hline \multirow{2}{*}{2011} & $\begin{array}{l}\text { Remodelación del edificio } \\
\text { ubicado en Rayón } 411\end{array}$ & $\begin{array}{l}\text { Mercado orgánico } \\
\text { «El Pochote» }\end{array}$ \\
\hline & $\begin{array}{l}\text { Implementación del proyecto } \\
« \text { Plan Luz» }{ }^{26}\end{array}$ & $\begin{array}{l}\text { Iluminación de edificios } \\
\text { históricos }\end{array}$ \\
\hline \multirow[b]{2}{*}{2014} & $\begin{array}{l}\text { Mejoramiento de imagen } \\
\text { de tres mercados: Benito Juárez, } \\
\text { La Merced y } 20 \text { de Noviembre }\end{array}$ & $\begin{array}{l}\text { Centro de abastecimiento } \\
\text { urbano }\end{array}$ \\
\hline & $\begin{array}{l}\text { Peatonalización de varias calles: } \\
\text { Cruz de Piedra-Carmen Alto; } \\
\text { García Vigil, tramo Allende- } \\
\text { Alameda de León }\end{array}$ & Turística o recreativa \\
\hline
\end{tabular}

Fuente: elaboración propia con datos de informes de H. Ayuntamiento de Oaxaca de Juárez (1976-2015).

Con la intención de recuperar la identidad y la cohesión en la sociedad el gobierno implementó programas culturales. El propósito era que los habitantes reconocieran y revalorizaran las costumbres y las tradiciones que caracterizan a sus barrios; no obstante, dicho acto tuvo más tintes turísticos que lo declarado. No se abordaron las problemáticas que enfrentaban los espacios en la manera de conectar gobierno y habitantes, todo quedó en la promoción de productos y festividades; en consecuencia, los beneficiarios fueron en su mayoría turistas y algunos comerciantes (cuadro 6).

${ }^{26}$ Iluminación arquitectónica de edificios históricos con la finalidad de llevar a Oaxaca a la categoría de Ciudad Luz. 


\author{
Cuadro 6 \\ Actividades culturales emprendidas \\ por el gobierno para recuperar la identidad
}

\begin{tabular}{c|lc} 
Año & \multicolumn{1}{|c}{ Programa } & Actores \\
\hline \multirow{2}{*}{2009} & Mi Oaxaca Linda $^{27}$ & Gobierno municipal \\
& Identidad de los barrios de Oaxaca & Gobierno municipal-Conaculta \\
2012 & Coro de la ciudad & Gobierno municipal \\
2014 & MP3 Oax-Flashmob & \\
\hline
\end{tabular}

Fuente: elaboración propia con datos de informes de H. Ayuntamiento de Oaxaca de Juárez (1976-2015).

En síntesis, las tareas efectuadas en el periodo 2006-2015 muestran una fuerte intervención en inmuebles, remodelación de calles y avenidas, ejecución de obras públicas y mejoramiento de imagen. Los informes de gobierno registran un total de 175 obras en esta orientación, mientras que la cantidad de acciones emprendidas para impulsar el turismo fue de 302. Al comparar con etapas anteriores, ${ }^{29}$ los datos indican que entre 1976-1986 se desarrollaron 44 obras y 35 actividades; entre 1987-2005 se contabilizaron 61 trabajos constructivos y 19 acciones culturales. Tales resultados evidencian un claro interés gubernamental por recuperar una dinámica turística que, conforme al discurso oficial, fue arrebatada a causa

27 Se desarrollaron 131 actividades: 79 culturales, 22 deportivas, 16 sociales y 14 de difusión. ${ }^{28}$ Proyecciones de videomapping sobre templos emblemáticos, como el convento de Santo Domingo de Guzmán, la Basílica de la Soledad, la iglesia Sangre de Cristo, por mencionar algunos.

${ }^{29}$ Con base en información recabada a la fecha se establecen tres momentos: 1976-1986, periodo anterior a la declaratoria en que se define por decreto presidencial la zona de monumentos; 1987-2005, etapa posterior a la declaratoria patrimonial, pero previa a los hechos sociales; 2006-2015, fase de recuperación. 
de los problemas sociales. Cabe preguntarse, ¿cómo evolucionó la afluencia turística en el tiempo?, ¿presenta crecimiento constante o es variable?, ¿existe una baja posterior a los hechos sociales en 2006 como señalan los discursos? Después de una década de los sucesos, irealmente se alcanzó el objetivo o se sigue construyendo?

\section{Una actividad económica volátil: el turismo}

Con relación a la dinámica turística en el centro de Oaxaca, se estudiaron los anuarios estadísticos del Instituto Nacional de Estadística y Geografía (INEGI), 1990-2015; las cifras indican una afluencia turística en constante crecimiento entre 1995 y 2005, que luego disminuyó entre 2006 y 2009, se recuperó e incluso rebasó la cifra récord establecida en 2004 (1 millón 2 mil 719 turistas) y se posicionó con 1 millón 148 mil 616 visitantes en 2015 (gráfica 1). La disminución que expone la dinámica obedece a procesos locales y nacionales. Según la Secretaría de Turismo (2013) dos situaciones determinaron el descenso de visitantes a la ciudad: los eventos de 2006 y la epidemia del virus gripal A (H1Nl) en 2009, acentuándose sobre todo en el turismo internacional. Sin embargo, no son los únicos hechos que exponen la vulnerabilidad de la actividad: entre 1993 y 1994 también se presentó baja afluencia debido a la crisis económica que atravesó el país. Las variaciones evidencian su fragilidad ante cualquier evento disruptor; ello cuestiona el interés gubernamental de apoyar al sector para posicionarlo como motor de crecimiento, ¿cuánto más podrá financiar su desarrollo con la intención de consolidarlo ante la crisis económica que atraviesa el país? 


\section{Gráfica 1}

Afluencia turística en Oaxaca de Juárez, 1990-2015

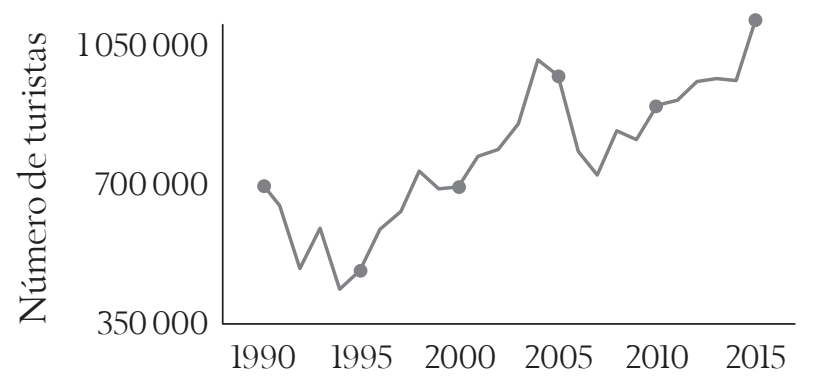

Fuente: elaboración propia con información de INEGI (1990-2015).

Se observa que la política turística emprendida por el gobierno posterior a los hechos de 2006 alcanzó su objetivo de recuperar la afluencia; no obstante es necesario inquirir en qué situación se encuentra el comercio enfocado al turismo, puesto que a la fecha la iniciativa privada y diversas asociaciones continúan denunciando la crisis que enfrentan.

El comportamiento del sector turístico en el lapso de 25 años en la ciudad de Oaxaca (1990-2015), se sintetiza en crecimiento en dos rubros: alojamiento y servicios de alimentos y bebidas; estabilidad en agencias de viajes (gráfica 2). En el caso del alojamiento se percibió una ligera disminución, así como la existencia de una capacidad subaprovechada ${ }^{30}$ por los problemas sociales acaecidos en 2006 que provocaron el cierre de varios negocios. Este argumento es sostenido por la Cámara de Hoteles y Moteles de Oaxaca, pero es cuestionable si se amplía el rango temporal del estudio. Pese al posible cierre de comercios por la falta de oferta, existe un

${ }^{30}$ En ese periodo la ocupación promedio no rebasó 45 por ciento. 
real aumento de establecimientos; asimismo, en añadidura a esa multiplicación de habitaciones, la demanda se modifica. Por lo tanto, los acontecimientos sociales no están intrínsecamente asociados a la actividad, puesto que no frenaron la expectativa empresarial.

Respecto al servicio de alimentos y bebidas, de 1994 a 2015 su comportamiento se mostró inestable, con incrementos y disminuciones; pero posterior a 2006 la cantidad de empresas con ese perfil se mantuvo. Para Sectur (2013) aquel fue el sector que más empleo generó en 2012, dentro de la gama de comercios vinculados al turismo; añade que enfrentó (y sigue enfrentando) el problema del ambulantaje, principalmente en el área del Centro Histórico, el cual se incrementa en temporadas altas y festividades, y ocasiona degradación y contaminación de la imagen visual, obstrucción de la vía pública y descontento por parte del comercio establecido (Sectur, 2013:69).

Por su parte, durante 1994-2015, las agencias de viajes presentaron un comportamiento estable. Sólo entre 2000-2003 se registraron aumentos, en 2002 se contabilizaron 57 establecimientos; pero después de 2004 el promedio se fijó en 45. Acción que contrasta con lo argumentado por los dueños de las agencias, quienes declaran que a raíz de los sucesos de 2006 el sector registró pérdidas y ello ocasionó el cierre de algunos establecimientos, así como readecuaciones de precios en otros en un afán por mantenerse en el mercado. Cabe subrayar que el crecimiento en el uso del internet para llevar a cabo reservaciones de todo tipo es una característica reciente de la actividad turística que influye en la cantidad y en el número de operaciones de las agencias en el mundo entero.

La información estadística evidencia una baja en la afluencia turística a causa de los problemas sociales, mas logra recuperarse una década después, incluso se incrementa. A la fecha los discursos emitidos por distintas 
asociaciones y organizaciones sociales se mantienen: Oaxaca sufre una crisis económica que debe solucionar el gobierno. Una recesión que se compagina con la registrada en el nivel estatal, la cual debe ser resuelta de manera integral y no sectorial. Según estadísticas y notas periodísticas en la entidad se incorporaron 173 trabajadores al día al mercado laboral informal en 2016, lo que significa un crecimiento de 11 por ciento anual (Silva, 2017). La referencia permite señalar que falta invertir en otros sectores; vivir del turismo conlleva riesgos económicos que difícilmente pueden solventarse a la larga.

\section{Gráfica 2}

Dinámica de los establecimientos asociados a la actividad turística en Oaxaca de Juárez, 1990-2015

$$
- \text { Alojamiento }-\begin{gathered}
\text { Alimentos } \\
\text { y bebidas }
\end{gathered}-\begin{array}{r}
\text { Agencias } \\
\text { de viajes }
\end{array}
$$

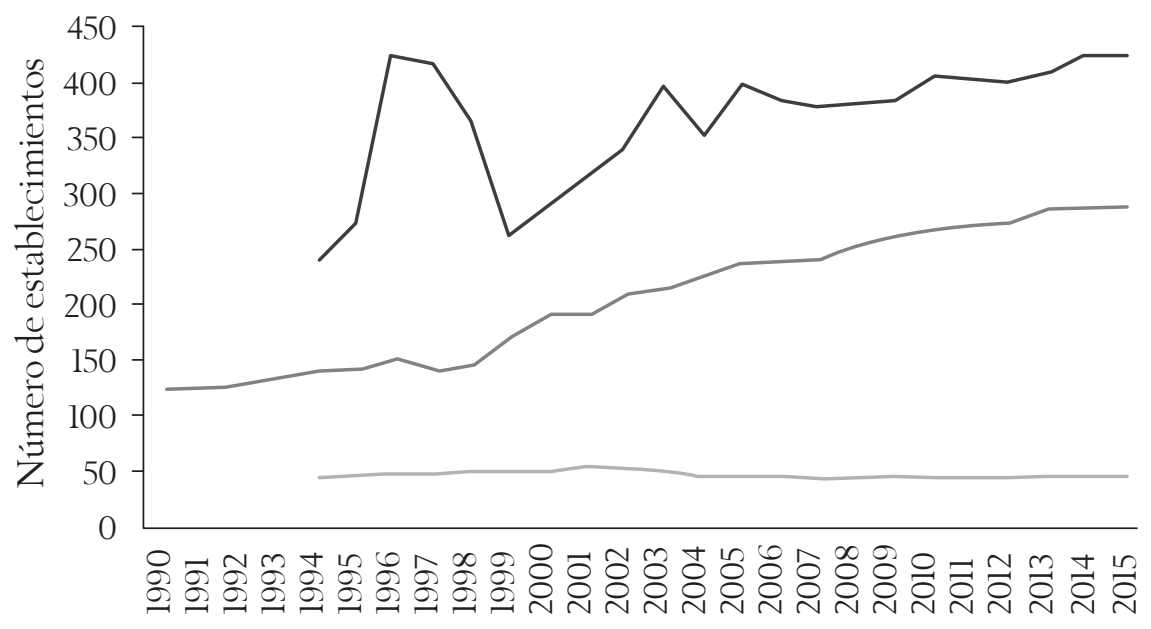

Fuente: elaboración propia con información de INEGI (1990-2015). 


\section{Efectos del despliegue turistificador en el Centro Histórico de Oaxaca}

Después de nueve décadas de fomento al turismo por parte de los gobiernos estatal y municipal, los efectos físicos y socioespaciales son palpables. Relativo a lo físico, la adaptación de la ciudad a las actividades comerciales o turísticas ha implicado cambios morfológicos, que en ocasiones vulneran la estructura arquitectónica. Un ejemplo es el exconvento San Pablo; en su etapa de rehabilitación se cuestionó el papel de las instituciones encargadas de resguardar el patrimonio, INAH y gobierno municipal, además de intelectuales como Francisco Toledo, por permitir y tolerar la sustitución de materiales y las modificaciones de interiores que efectuó la FAHHO (Valdivia, 2012). De igual forma, los inmuebles ubicados en el primer cuadro de la ciudad registran alteraciones en su arquitectura; su adaptación constante a las actividades imperantes de cada época ha vulnerado su estructura, por tanto, quedan en la actualidad pocos vestigios originales (figura l).

Figura 1

Portal de mercaderes, 1909 y 2017
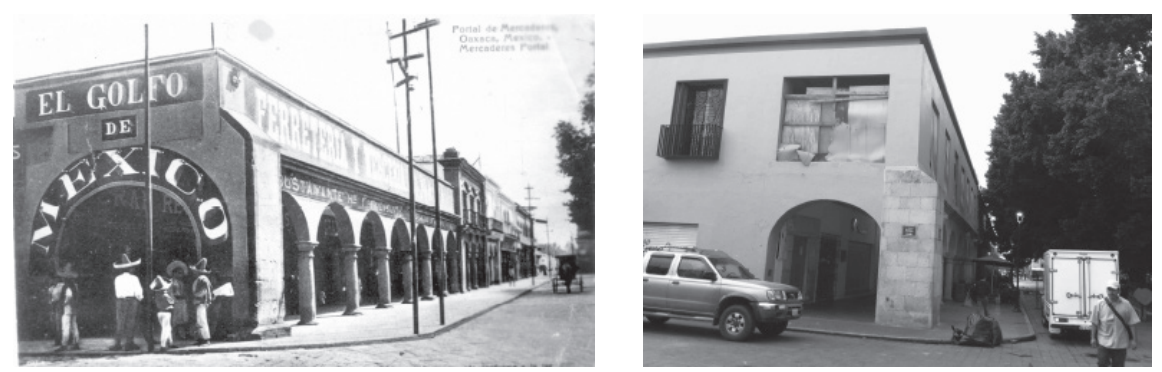

Fuente: Fotografía Velázquez y recorridos de campo. 


\section{La tURistificación en el Centro Histórico de OAXACA}

Difícilmente los dueños de inmuebles que aún conservan su función habitacional logran rehabilitarlos o adecuarlos. Miembros del Colegio de Valuadores del Estado de Oaxaca señalaron en entrevista que la reglamentación se muestra inflexible ante los intereses de los habitantes. En algunos casos sus solicitudes fueron rechazadas, en uno de ellos porque debían emplear materiales originales y eso generaba aumento de costos constructivos, difíciles de absorber por propietarios con recursos mínimos; en otro caso, se tenía la intención de ampliar una cochera para facilitar el traslado de una persona de la tercera edad. En general, se observa una ambivalencia reglamentaria: por un lado, poca flexibilidad en la adaptación de inmuebles con usos habitacionales; por otro lado, una abierta disposición en adecuaciones para fines comerciales, sean turísticos o tradicionales. Este proceder puede afectar el sentido de Centro Histórico: de caracterizarse como centro patrimonial vivo se transformaría en un espacio marcado por la museificación, como lo advierte la literatura para otros espacios que experimentan avanzados procesos de despoblamiento, se volvería un parque temático turístico.

Otro factor que vulnera la existencia de los inmuebles históricos y en general las edificaciones que componen la ciudad de Oaxaca son los sismos que ocurren periódicamente, además de la ausencia de políticas preventivas y de recursos para su rescate y mantenimiento, como se evidenció en septiembre de 2017.

La importancia de la conservación de inmuebles históricos no sólo radica en su atractivo turístico o incluso en la necesidad de preservar el estatuto de Patrimonio de la Humanidad, que le fue otorgada por la UNESCO en 1987, sino en el hecho de que sintetiza parte de una identidad y cultura oaxaqueña. En ese nivel, cabe plantearse si la disyuntiva es determinar qué proceso tiene más peso: una recuperación de inmuebles según intereses específicos 
o un abandono, lo cual aceleraría su deterioro. Su permanencia implica que juntos, gobierno, sector privado y sociedad, construyan un proyecto preventivo y una reglamentación concientizada, por mencionar algunos puntos, con el fin de contrarrestar el permanente deterioro y vulnerabilidad que padece el patrimonio.

Relativo al aspecto socioespacial, son tres los efectos identificados hasta el momento a causa del despliegue turistificador en el Centro Histórico de Oaxaca: expulsión de población tradicional residente; reemplazo de población por nuevos ocupantes; y modificación en el uso del espacio público. Mediante entrevistas se obtuvo que el origen de tales efectos se asocia con la ubicación de los inmuebles y sus valores de renta y venta, los cuales impactan de manera directa en su uso habitacional o comercial, y en general en la dinámica poblacional y económica del área. La existencia de un escaso mercado de suelo para ofertar detona en la elevación de los precios de los inmuebles, ${ }^{31}$ por lo que no extraña a los promotores inmobiliarios que la ciudad presente los precios de venta en edificaciones más caros del país (Sánchez, 2017). Podría suponerse que los problemas sociales que atraviesa el estado, como el conflicto de 2006 (que una década después se reactivó mediante bloqueos, plantones, marchas, entre otras formas de protestas) revertirían la tendencia al alza de los precios inmobiliarios, pero los datos exponen sólo una desaceleración temporal en el crecimiento de las plusvalías. ${ }^{32}$

Entender la dinámica de precios de alquileres inmobiliarios en la ciudad implica realizar un comparativo de precios según usos, habitacional y comercial, para constatar la situación compleja que enfrenta la vivienda

${ }^{31}$ En la ciudad existen dos tipos de propiedad, la privada que representa 30 por ciento del total; y la social, llámese ejidal o comunal, que concentra la mayor parte, 70 por ciento del suelo.

32 Actualmente dicho crecimiento se encuentra en proceso de estabilización, se posiciona entre 10 y 12 por ciento anual. 


\section{LA tURistificación en el Centro Histórico de OAXaca}

del centro. Se observa un diferencial en la ganancia que va de 50 a 100 por ciento cuando una edificación se destina a uso comercial; ${ }^{33}$ ello disuade a los propietarios de los inmuebles al uso habitacional y, en consecuencia, incita un proceso de expulsión poblacional.

De los inmuebles que aún cuentan con uso habitacional, según información referida en la entrevista, su conservación depende de los objetivos de sus propietarios o de los posibles compradores, interviene también la ubicación del inmueble. ${ }^{34}$ Los potenciales compradores son en su mayoría comerciantes, su finalidad es invertir en el área o expandir su negocio; en menor medida los mismos residentes efectúan la compra con el propósito de permanecer en la zona; el tercer lugar lo ocupan los artistas y extranjeros. En el caso de los artistas, el deseo de insertarse en el mercado del arte, actualmente en boga en Oaxaca, incita a un cambio de residencia. Por su parte, los extranjeros, a quienes atrae el ambiente patrimonial y cultural de la ciudad, aunado a las ganancias obtenidas debido al cambio de divisas, se ven beneficiados al invertir en determinado bien; integran este perfil las personas de la tercera edad, jubilados, de nacionalidad estadounidense o canadiense. La inserción de nuevos residentes a la zona llama la atención de valuadores, no sólo por el perfil de la población que llega al centro, sino también porque su actuar impacta en las plusvalías de la zona. ${ }^{35} \mathrm{La}$ compra

${ }^{33}$ Para uso habitacional de un departamento de tamaño medio en una zona promedio, un propietario recauda entre 2 y 3 mil pesos mensuales; para uso comercial de un local pequeño en un sector promedio, el dueño percibe entre 4 a 6 mil pesos mensuales. Cuando los inmuebles se ubican en corredores urbanos, los precios pueden duplicarse o incluso triplicarse.

${ }^{34}$ La proximidad de algún corredor urbano a la edificación determinará su destino. Oficialmente la agencia centro registra 13 corredores urbanos de primer orden y ocho de segundo orden; el 80 por ciento de ellos se ubica en el Centro Histórico de Oaxaca.

${ }^{35}$ Compran al costo que oferta la población, los precios del mercado inmobiliario no intervienen totalmente en la transacción: la ubicación, el estado del inmueble, las mejoras de imagen, entre otros elementos, son factores primordiales para adquirir un bien. 
resulta sobrevaluada, lo que coarta las posibilidades de la población local en adquirir un bien. ${ }^{36}$ Con base en lo anterior se advierte un reemplazo de población tradicional por otra de nivel socioeconómico mayor, sin que hasta el momento se confirme la existencia de un vasto proceso de gentrificación.

La distribución espacial del comercio en el Centro Histórico de Oaxaca obedece a la ejecución de políticas urbanas desarrolladas por los gobiernos estatal y municipal durante décadas. El énfasis en el mejoramiento de imagen de ciertos sectores y la definición de corredores urbanos, por mencionar algunas acciones, estimularon la producción de zonas comerciales delimitadas según sus funciones tradicionales o turísticas. Los valores de renta o venta de inmuebles no intervienen en su destino, en ambos usos los costos de oferta son equiparables. ${ }^{37}$ Por ejemplo, si se parte de la Alameda hacia el noreste el destino es netamente turístico; y del zócalo hacia el suroeste su ocupación es tradicional. Esta dinámica impacta la producción del espacio, definido por ofertas comerciales y gobierno. Población o residentes tenderán a recorren áreas tradicionales para realizar sus actividades cotidianas; mientras que los turistas optarán por espacios destinados al entretenimiento y al ocio. Asimismo, debido a la sucesiva toma del zócalo por distintas organizaciones sociales, recientemente el gobierno modificó la ruta del desfile delegacional que abre las festividades de la Guelaguetza, lo que altera los recorridos turísticos tradicionales; la acción permite cuestionar el

\footnotetext{
${ }^{36}$ Se supo de la venta de una casa en cinco millones y medio de pesos a un extranjero. En el mercado inmobiliario esa edificación no rebasaba en su momento los tres millones, por el estado en que se encontraba y las dimensiones que registraba (valuador inmobiliario, 2017). ${ }^{37}$ En las calles de Las Casas, Trujano, 20 de Noviembre, Aldama, con enfoque tradicional, las rentas por un local de $9 \mathrm{~m}^{2}$ van de 10 a 30 mil pesos; mientras que en las calles de García Vigil, Macedonio Alcalá, 5 de Mayo, con perspectiva turística, las rentas por un local de $12 \mathrm{~m}^{2}$ fluctúan entre 20 y 40 mil pesos.
} 


\section{La tURistificación en el Centro Histórico de OAXACA}

propio uso de los espacios que ahora se integran al desarrollo de ese tipo de celebraciones.

Como se advierte, no sólo la dinámica turística incide en el desarro-

1lo de transformaciones socioespaciales en el Centro Histórico de Oaxaca, también lo hace el comercio tradicional. Quienes han modelado este proceso segregativo a través de obras y acciones desarrolladas durante décadas son los gobiernos estatal y municipal. Cabría analizar las mutaciones surgidas por la llegada de nuevos pobladores a la zona: ¿se integran a las tradiciones barriales?, ¿cuáles son sus actividades cotidianas?

\section{Conclusiones}

Oaxaca enfrenta problemas sociales, políticos y económicos que no pueden resolverse mediante el impulso turístico. Lo sucedido en 2006 fue un hartazgo social desencadenado por el pésimo actuar gubernamental en la solución de problemas. Salir de esa situación implicaría una voluntad política de la presente y de las futuras administraciones gubernamentales, pero principalmente una continuidad en el desarrollo de las políticas públicas con visión integral.

Si bien el turismo es una actividad que aporta al crecimiento económico de la entidad, no es la principal; la mayoría de los municipios vive del sector primario. La concentración de recursos económicos en el fomento turístico, sumada al esfuerzo dedicado a la construcción de discursos para legitimar las acciones gubernamentales ante la sociedad, ahonda las desigualdades urbanas y sociales, además de afectar las finanzas; a la fecha persiste un fuerte endeudamiento estatal. 
A escala local, el desarrollo de la actividad turística y tradicional ha generado transformaciones urbanas y socioespaciales, aún no evaluadas por el gobierno, lo cual plantea incertidumbre sobre el futuro patrimonial y la propia habitabilidad de la zona. Los cambios de usos que presenta la mayor parte de las edificaciones afectan no sólo su estructura arquitectónica, también los modos de vida de la población que todavía reside en el espacio. Este proceso no es cuestionado entre los servidores públicos que tienen a su cargo el resguardo o conservación de los inmuebles, lo que denota una normatividad laxa a conveniencias. Sin embargo, el gobierno, con la finalidad de exhibir una ciudad tranquila y ordenada, implementa soluciones parciales para contrarrestar la baja afluencia de visitas a la ciudad: obras de embellecimiento, desalojos temporales de comercio informal en temporadas altas o en ocasión de eventos masivos, modificación de rutas turísticas en el desarrollo de festividades, entre otras acciones que a la larga le significan costos políticos, sociales y económicos aún no analizados.

Es tarea de las administraciones estatal y municipal hacer frente al juego de intereses que desarrollan las organizaciones sociales y los sindicatos, si se desea alcanzar el objetivo de mantener o aumentar la cantidad de visitantes a la zona, puesto que la imagen de la ciudad está de por medio. Es imprescindible garantizar la tranquilidad y el disfrute del espacio no sólo al turismo, también a la población local y los aún residentes, ante los constantes bloqueos de calles y plantones. La amenaza o concreción en la toma de espacios públicos plantea una baja capacidad de gestión existente entre ambos niveles de gobierno, estatal y municipal, en la solución de problemas, así como una posible complicidad o pago de favores a ese tipo de organizaciones, temas pendientes a estudiar. 


\section{Referencias}

Bertoncello, Rodolfo (2006), «Turismo, territorio y sociedad. El «mapa turístico de la Argentina»», en Amalia Inés Geraiges de Lemos, Mónica Arroyo y María Laura Silveira (eds.), América Latina: cidade, campo e turismo, Brasil, Consejo Latinoamericano de Ciencias Sociales, pp. 317-335.

Córdoba y Ordóñez, Juan (2009), «Turismo, desarrollo y disneyzación: ¿una cuestión de recursos o de ingenio?, Investigaciones Geográficas (70), pp. 3354, en http://www.redalyc.org/articulo.oa?id=56912238003

De Sicilia Muñoz, Alejandrina (2012), «Evaluación económica del impacto del turismo cultural en la ciudad de Zacatecas», Investigaciones Geográficas, Boletín del Instituto de Geografía (78), pp. 89-103, en http://www.redalyc.org/ articulo.oa? $\mathrm{id}=56924410008$

Del Valle Guerrero, Ana Lía y Silvana Soledad Gallucci (2015), «Aporte teórico conceptual al turismo como disciplina académica a partir de la patrimonialización como proceso de valorización turística de los territorios», PASOS, 13(1), pp. 145-156, en http://bddoc.csic.es:8080/detalles.html;jsessionid=5F589B33AC6325B565487EC57E24B556?id=776636\&bd=ISO\&tabla=docu

Emeequis (agosto de 2016), «Harp Helú llama a empresarios, CNTE y gobierno a actuar positivamente», Emeequis, en http://www.nvinoticias.com/nota/25161/ harp-helu-llama-empresarios-cnte-y-gobierno-actuar-positivamente.

García, Ismael (1 de marzo de 2017), «Desmantelan Museo del Palacio de Gobierno en Oaxaca», El Universal, en http://www.eluniversal.com.mx/articulo/ estados/2017/03/1/desmantelan-museo-del-palacio-de-gobierno-en-oaxaca

Gobierno del Estado de Oaxaca (28 de abril de 2011), «Se recupera edificio de avenida Juárez como Casa Oficial de Gobierno», Quadritín, en https:// 


\section{MABel Yescas SÁNCHEZ}

oaxaca.quadratin.com.mx/Se-recupera-edificio-de-Avenida-Juarez-comoCasa-Oficial-de-Gobierno/

González Hernández, Guadalupe Margarita (2014), Circo sin pan. Regeneración y mercantilización en el Centro Histórico, Ciudad de México, Universidad Autónoma de Zacatecas/Miguel Ángel Porrúa.

Hiernaux-Nicolas, Daniel y Carmen Imelda González Gómez (2015), «Patrimonio y turismo en centros históricos de ciudades medias. ¿Imaginarios encontrados?», URBS, 5(2), pp. 111-125, en http://www2.ual.es/urbs/index.php/urbs/ article/view/hiernaux_gonzalez/253

(2014), «Turismo y gentrificación: pistas teóricas sobre una articulación», Revista de Geografía Norte Grande (58), pp. 55-70, en http://www.redalyc. org/pdf/300/30031739004.pdf

H. Ayuntamiento de Oaxaca de Juárez (1976), Primer informe de gobierno, Oaxaca de Juárez, Cabildo municipal.

(1977), Segundo informe de gobierno, Oaxaca de Juárez, Cabildo municipal. (1980), Primer informe de gobierno, Oaxaca de Juárez, Cabildo municipal. (1982), Segundo informe de gobierno, Oaxaca de Juárez, Cabildo municipal. (1984), Primer informe de gobierno, Oaxaca de Juárez, Cabildo municipal. (1985), Segundo informe de gobierno, Oaxaca de Juárez, Cabildo municipal. (1986), Tercer informe de gobierno, Oaxaca de Juárez, Cabildo municipal. (1987), Primer informe de gobierno, Oaxaca de Juárez, Cabildo municipal. (1989), Segundo informe de gobierno, Oaxaca de Juárez, Cabildo municipal. (1990), Primer informe de gobierno, Oaxaca de Juárez, Cabildo municipal. (1991), Segundo informe de gobierno, Oaxaca de Juárez, Cabildo municipal. (1993), Primer informe de gobierno, Oaxaca de Juárez, Cabildo municipal. (1994), Segundo informe de gobierno, Oaxaca de Juárez, Cabildo municipal. (1995), Tercer informe de gobierno, Oaxaca de Juárez, Cabildo municipal. 


\section{La tURistificación en el Centro Histórico de OAXACA}

(1996), Primer informe de gobierno, Oaxaca de Juárez, Cabildo municipal. (1997), Segundo informe de gobierno, Oaxaca de Juárez, Cabildo municipal. (1998), Tercer informe de gobierno, Oaxaca de Juárez, Cabildo municipal. (1999), Primer informe de gobierno, Oaxaca de Juárez, Cabildo municipal. (2000), Segundo informe de gobierno, Oaxaca de Juárez, Cabildo municipal. (2001), Tercer informe de gobierno, Oaxaca de Juárez, Cabildo municipal. (2002), Primer informe de gobierno, Oaxaca de Juárez, Cabildo municipal. (2003), Segundo informe de gobierno, Oaxaca de Juárez, Cabildo municipal. (2004), Tercer informe de gobierno, Oaxaca de Juárez, Cabildo municipal. (2005), Primerinforme de gobierno, Oaxaca de Juárez, Cabildo municipal. (2006), Segundo informe de gobierno, Oaxaca de Juárez, Cabildo municipal. (2007), Tercer informe de gobierno, Oaxaca de Juárez, Cabildo municipal. (2008), Primerinforme de gobierno, Oaxaca de Juárez, Cabildo municipal. (2009), Segundo informe de gobierno, Oaxaca de Juárez, Cabildo municipal. (2010), Tercer informe de gobierno, Oaxaca de Juárez, Cabildo municipal. (2011), Primer informe de gobierno, Oaxaca de Juárez, Cabildo municipal. (2012), Segundo informe de gobierno, Oaxaca de Juárez, Cabildo municipal. (2013), Tercer informe de gobierno, Oaxaca de Juárez, Cabildo municipal. (2014), Primer informe de gobierno, Oaxaca de Juárez, Cabildo municipal. (2015), Segundo informe de gobierno, Oaxaca de Juárez, Cabildo municipal.

Instituto Nacional de Estadística y Geografía (1986), Anuario estadístico: Oaxaca, en http://www.beta.inegi.org.mx/app/publicaciones/

(1987) Anuario estadístico: Oaxaca, en http://www.beta.inegi.org.mx/ app/publicaciones/

(1988), Anuario estadístico: Oaxaca, en http://www.beta.inegi.org.mx/ app/publicaciones/ 


\section{MABel Yescas SÁNCHEZ}

(1989), Anuario estadístico: Oaxaca, en http://www.beta.inegi.org.mx/ app/publicaciones/

(1990), Anuario estadístico: Oaxaca, en http://www.beta.inegi.org.mx/ app/publicaciones/

(1991), Anuario estadístico: Oaxaca, en http://www.beta.inegi.org.mx/ app/publicaciones/

(1992), Anuario estadístico: Oaxaca, en http://www.beta.inegi.org.mx/ app/publicaciones/

(1993), Anuario estadístico: Oaxaca, en http://www.beta.inegi.org.mx/ app/publicaciones/

(1994), Anuario estadistico: Oaxaca, en http://www.beta.inegi.org.mx/ app/publicaciones/

(1995), Anuario estadístico: Oaxaca, en http://www.beta.inegi.org.mx/ app/publicaciones/

(1996), Anuario estadístico: Oaxaca, en http://www.beta.inegi.org.mx/ app/publicaciones/

(1997), Anuario estadístico: Oaxaca, en http://www.beta.inegi.org.mx/ app/publicaciones/

(1998), Anuario estadístico: Oaxaca, en http://www.beta.inegi.org.mx/ app/publicaciones/

(1999), Anuario estadistico: Oaxaca, en http://www.beta.inegi.org.mx/ app/publicaciones/

(2000), Anuario estadístico: Oaxaca, en http://www.beta.inegi.org.mx/ app/publicaciones/

(2001), Anuario estadístico: Oaxaca, en http://www.beta.inegi.org.mx/ app/publicaciones/ 


\section{La turistificación en el Centro Histórico de OAXaca}

(2002), Anuario estadístico: Oaxaca, en http://www.beta.inegi.org.mx/ app/publicaciones/

(2003), Anuario estadístico: Oaxaca, en http://www.beta.inegi.org.mx/ app/publicaciones/

(2004), Anuario estadístico: Oaxaca, en http://www.beta.inegi.org.mx/ app/publicaciones/

(2005), Anuario estadístico: Oaxaca, en http://www.beta.inegi.org.mx/ app/publicaciones/

_ (2006), Anuario estadístico: Oaxaca, en http://www.beta.inegi.org.mx/ app/publicaciones/

(2007), Anuario estadístico: Oaxaca, en http://www.beta.inegi.org.mx/ app/publicaciones/

(2008), Anuario estadístico: Oaxaca, en http://www.beta.inegi.org.mx/ app/publicaciones/

(2009), Anuario estadistico: Oaxaca, en http://www.beta.inegi.org.mx/ app/publicaciones/

(2010), Anuario estadístico: Oaxaca, en http://www.beta.inegi.org.mx/ app/publicaciones/

(2011), Anuario estadístico: Oaxaca, en http://www.beta.inegi.org.mx/ app/publicaciones/

(2012), Anuario estadístico: Oaxaca, en http://www.beta.inegi.org.mx/ app/publicaciones/

(2013), Anuario estadístico: Oaxaca, en http://www.beta.inegi.org.mx/ app/publicaciones/

(2014), Anuario estadístico: Oaxaca, en http://www.beta.inegi.org.mx/ app/publicaciones/ 


\section{MABEL Yescas SÁNCHEZ}

(2015), Anuario estadistico: Oaxaca, en http://www.beta.inegi.org.mx/ app/publicaciones/

Lira Vásquez, Carlos (2014), «El discurso patrimonial: una coartada para frenar el desarrollo de la ciudad de Oaxaca», Urbano (29), pp. 72-87, en http://revistas. ubiobio.cl/index.php/RU/article/view/238

Martínez Mauri, Mónica (2015), «Una mirada sobre la turistificación de la antropología del desarrollo en el Estado español», PASOS, 13(2), pp. 347-358, en https://dialnet.unirioja.es/servlet/articulo?codigo=5089494

Martínez Vásquez, Víctor Raúl (2008), «Crisis política y represión en Oaxaca», El Cotidiano, 23(148), pp. 45-62, en http://www.redalyc.org/pdf/325/32514805.pdf Mateo, Ivonne (25 de agosto de 2017), «Falso altruismo y cacicazgo cultural de la Fundación Alfredo Harp Helú», Díaz de Juárez, en http://diazdejuarez. com/falso-altruismo-cacicazgo-cultural-la-fundacion-alfredo-harp-heluoaxaca/

Navarrete Escobedo, David (2017), «Turismo gentrificador en ciudades patrimoniales. Exclusión y transformaciones urbano-arquitectónicas del patrimonio en Guanajuato, México», Revista INVI, 32(89), pp. 61-83, en http://revista invi.uchile.cl/index.php/INVI/article/view/1019

Pérez Winter, Cecilia (2013), «Patrimonialización, turistificación y autenticidad en Exaltación de la Cruz, Argentina», Estudios y perspectivas en turismo, 22(4), pp. 785-804.

Sánchez, Virgilio (mayo de 2017), «Oaxaca, con las casas más caras del país; incluso, más que frente al mar», Noticias Voz e Imagen, en http://www.nvinoticias. com/nota/59287/vivienda-mas-cara-en-oaxaca-que-en-paraiso-frente-al-mar Secretaría de Turismo (2013), Agendas de competitividad de los destinos turísticos de México: Oaxaca, en http://www.sectur.gob.mx/wp-content/uploads /2015/02/PDF-Oaxaca.pdf 


\section{La tURistificación en el Centro Histórico de OAXACA}

Silva, Pedro (20 de febrero de 2017), «Crea Oaxaca un ambulantaje cada ocho minutos», Noticias Voz e Imagen, en http://www.nvinoticias.com/nota/51738 /trabajan-en-la-informalidad-mas-de-600-mil-oaxaquenos

Sorroza Polo, Carlos (2008), «La crisis política de Oaxaca: componentes, alcances y propuestas de salida», El Cotidiano 23(148), pp. 21-36, en http://www. redalyc.org/articulo.oa?id=32514803

Troncoso, Claudia A. y Analía V. Almirón (2005), «Turismo y patrimonio. Hacia una relectura de sus relaciones», Aportes y Transferencias 1, pp. 56-74, en http://www.redalyc.org/pdf/276/27690104.pdf

Valdivia, Sofía (15 de mayo de 2012), «Investiga UNESCO daño patrimonial de exconvento de San Pablo por el filántropo Alfredo Harp», Foro Político, en http://fpnoticias.com/2012/05/investiga-unesco-dano-patrimonial-de-exconvento-de-san-pablo-por-el-filantropo-alfredo-harp/ 